convenient means of strongly magnifying fliorescence, this fluorescence is not proof of the presence of mineral or rosin oil.

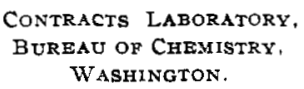

\section{NITRIC NITROGEN IN MIXED FERTILIZERS.}

By S. S. Peck.

Received September 5, 1911 .

At the 25th annual convention of the Association of Official Agricultural Chemists, the referee on the determination of nitrogen devoted his report to the determination of nitrogen in mixtures containing nitrate of soda. In neglecting the instructions of I 906 , he apologized by saying: ${ }^{\mathrm{I}}$

"Another phase of the nitrogen question considered last year and discussed in correspondence with the National Fertilizer Association seemed to outweigh in importance and urgency all others, namely, the determination of total nitrogen in mixed fertilizers to which nitrate of soda is added." In accordance with his instructions a number of determinations were made by the modified Gunning and Kjeldahl methods of mixtures, in which the source of nitrate was a solution of nitric acid, accurately standardized. The results of the different analysts showed wide variations, in the words of the author "mostly impossible." It seems very probable that the divergences were due to the heat generated by the acid and water, and Trescot expressed in his report to the referee his conviction that on dry samples, the modified Gunning method gives correct results.

In the Hawaiian Islands, about 40,000 tons of fertilizer are used annually, a large proportion of which . consists of high-grade mixtures containing from ' 8 to ro per cent. of nitrogen from ammonium sulphate, nitrate of soda, and organic sources, principally blood or tankage. The reliability of the modified Gunning or Kjeldahl methods is therefore of particular interest to both the fertilizer companies supplying the material and this Experiment Station, where samples of a greater part of the shipments to the plantations are analyzed. In the latter part of last year, complaints were received from a fertilizer company that even when the utmost care was observed in compounding mixtures containing nitrate, ammonia, and organic nitrogen, the analytical figures of both the company chemist and this station frequently showed a deficiency in this element. Two possible causes suggested themselves to us: first, the presence of too much moisture, and the imperfection of the Gunning method under this condition, and second, the loss of nitric nitrogen, as suggested by C. S. Cathcart. ${ }^{2}$ An investigation bearing on this point was started, according to the following plan: Mixtures were made containing 3 per cent. each of nitrogen from nitrate of soda, C. P., sulphate of ammonia, C. P., and high-grade tankage, along with superphosphate and potash salts. The nitrate of soda and suiphate of ammonia were powdered and dried; the tankage was screened through a $40-$ mesh sieve,

${ }^{1}$ Bull. 122, Bureau of Chemistry. U. S. Department of Agriculture.

2 This JoUrnal, Jan., 1911. only that part passing readily being used; the potash was added in one series as sulphate, in a second as muriate. The mixture contained about 8 per cent. of potash and 7.8 per cent. of phosphoric oxide soluble in water, in addition to the phosphoric oxide in the tankage, which was not determined. The ingredients were thoroughly mixed and portions weighed out as follows: Six ro-gram samples on watch glasses, and twenty-eight 2.5 -gram samples on paraffined paper, on watch glasses, half of each lot containing the potash as sulphate and half as muriate. The weights of all being recorded, one each of the rogram and two each of the 2.5 -gram lots were analyzed at once. The remainder were set aside for various intervals of time, being covered with large beakers so as to prevent contamination by dust or insects, but not hindering the free entrance of air. A further portion of each series was used for the determination of moisture according to the official method.

The 2.5-gram samples were analyzed at intervals by the modified Gunning method with the addition of 0.7 gram of mercury. A remarkable irregularity was obtained in our nitrogen figures, which we were finally able to trace to the imperfect manner in which the samples on the paraffined papers were covered by the salicylic-sulphuric acid mixture. A second set of determinations was started, with more regular results. The final figures in both sets, however, lead to the same conclusions.

The ten-gram samples were analyzed as follows: The sample was washed into a beaker and stirred in water for about an hour. It was then filtered onto a S. \& S. paper, the nitrogen in a similar lot of which having been previously determined and found to be negligible in quantity. The entire sample having been transferred to the paper, it was washed until the entire leadings amounted to $500 \mathrm{cc}$. The residue was then dried at a low temperature, and digested with mercury, sulphuric acid, and sulphate of potash. The nitrogen constituted the residual organic nitrogen.

Twenty-five cc. portion of the leadings were respectively distilled with caustic soda for ammonia nitrogen, and reduced with iron and sulphuric acid for the vitric and ammonia nitrogen, according to the Ulsch-Striet method. A like quantity was pipetted into a $500 \mathrm{cc}$. Kieldahl digestion flask, treated with iron and sulphuric acid to reduce the nitric nitrogen to ammonia, and then digested with sulphuric acid, mercury and potassium sulphate according to the usual Kjeldahl method. Considerable difficulty is met here, since anhydrous salts or double salts of iron as sulphate are precipitated and caust considerable bumping. The digestion is completed in about two hours, as there is really very little organic sitrogen present. Distillation presented the same difficulties as regards bumping, until we resorted to the ust of copper distilling flasks, since when we have had no other trouble except the uncertainty as to when the large quantity of acid present is neutralized by the soda lye. The distillate represents tie nitrogen as ammonium, nitrate and soluble organic; this added to the residual organic gives the total nitrogen. One 
error which enters into our results is that in distilling both for ammonia nitrogen and in the U1sch-Street method, using soda-lye; a certain amount of organic nitrogen is recorded as ammonia nitrogen. This, as will be shown later, amounts to only a very small percentage. Another error for which we have not corrected is the nitrogen in a blank. This may amount to as much as o.I per cent. in a 9 per cent. sample, but this correction has not been made since it in no wise interferes with our results used from a comparative standpoint; the difference must be the same in all determinations.

The results of our analyses are tabulated herewith:

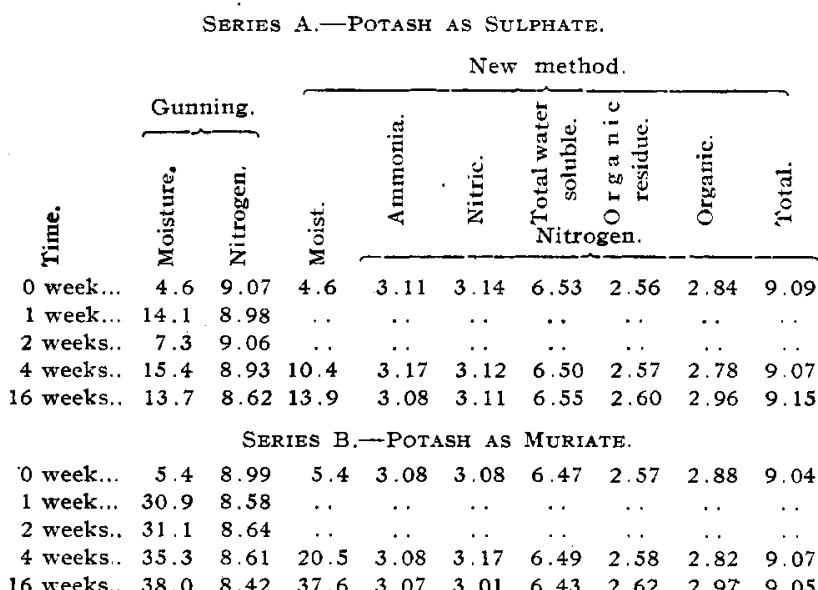

The moisture figures are given as percentages on dry matter. The moisture in the original sample was determined by drying; that in the other samples was calculated from the increase in weight. The percentages of nitrogen are reported in terms of the original material.

Series B increased in moisture considerably more than Series $A$, and there is a greater apparent loss in nitrogen, on the basis of the analyses by the Gunning method. The other method, however, shows that this loss is only apparent, and due to the imperfections of the Gunning method when applied to wet material. Each of the Gunning figures is the average of three determinations, the restults of which were fairly concordant.

The average of the six determinations by the new method gives the following results:

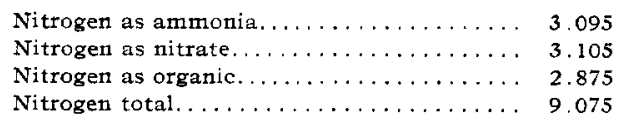

In a separate determination with the tankage alone, it was found that on distilling that soluble in water with soda-lye in the usual amounts employed, there was recovered as ammonia, nitrogen amounting to 2.8 per cent. of the total nitrogen in the material. This would necessitate a correction in the above figures of 0.08 per cent. in the ammonia, making a true average result of 3 .0 I 5 per cent. nitrogen as ammonia and 2.955 per cent. organic nitrogen.

In addition to these determinations, fertilizer samples showing a deficiency in total nitrogen, and containing the three forms of this element, have been re- analyzed by both the modified Gunning (with the addition of mercury) and the new method. In these instances, with one exception, the phosphoric oxide was present as insoluble lime phosphate, and in all, the potash was in the form of sulphate.

The results were as follows:

$\begin{array}{rccc}\text { Moisture. } & \text { Gunning. } & \text { New method. } & \text { Guarantee. } \\ 6.41 & 8.82 & 8.86 & 9 \\ 7.58 & 8.93 & 8.95 & 9 \\ 9.08 & 9.63 & 9.74 & 10 \\ 13.58 & 9.69 & 9.97 & 10 \\ 8.65 & 8.37 & 8.69 & 9 \\ 11.69 & 9.48 & 9.75 & 10 \\ 7.39 & 7.90 & 7.89 & 8\end{array}$

We interpret our results to signify that in the presence of sufficient moisture, the modified Gunning method gives incorrect results, and that a mixed fertilizer containing nitrate of soda and acid phosphate does not even after a lapse of four months lose any nitrogen. On arriving at this last'conclusion, we believe that we have avoided two errors inherent in Cathcart's method of investigation: (I) the unreliability of the modified Gunning method in the presence of much moisture, and (2) the uncertainty of moisture determinations in such a complex as a mixed fertilizer, with the unreliability of such a basis for comparative purposes, a point which has already been emphasized in ThIs JOURNAL. ${ }^{\mathbf{1}}$.

The larger part of the analytical work performed in obtaining data for this article was made by $\mathrm{Mr}$. A. E. Jordan.

Chemical Laboratory,

EXPERIMENT STATION, HAWAIIAN SUGAR

Planters' Association, Honolulu, '1. H.

\section{THE OIL OF DOUGLAS FIR: A PRELIMINARY STUDY OF ITS COMPOSITION AND PROPERTIES. \\ By H. K. BENSON AND MARC DARRIN. Received September 21, 1911}

In the steam distillation of resinous Douglas Fir wood, a crude greenish yellow turpentine of syrupy consistency is obtained. In the distillation process as practiced in the Pacific Northwest, steam under a boiler pressure of 45 to 60 abs. is used, and the retorts are usually placed under a diminished pressure at the end of the distillation period for the purpose of removing the last traces of oil from the wood chips.

The yield of this crude turpentine per cord is dependent upon the degree of pitchiness or "fatness" of the wood, but in the case of selected mill waste and stump wood, it ranges from 5 to 7 gallons per cord. When redistilled under low pressure steam, this yields from 4 to 5 gallons of a water-white turpentine which has a good odor and complies with the requirements for spirits of turpentine as established by the $U$. $\mathrm{S}$. Bureau of Chemistry in Bulletin rog, revised, Bureau of Chemistry, U. S. Dept. of Agriculture.

The residue after the removal of the turpentine consists of a clear viscous, yellow oil, similar in appearance to the pine oil obtained from the refining of the steam turpentine from Norway and of Michigan and long leaf pine of the south.

1 October, 1909, Editorial. 